Research Article

\title{
Multi-dimensional Chebyshev polynomials: a non-conventional approach
}

\author{
Clemente Cesarano ${ }^{1 *}$ \\ ${ }^{1}$ Section of Mathematics, International Telematic University UNINETTUNO, Roma, Italy \\ *Email address for correspondence: c.cesarano@uninettunouniversity.net
}

Communicated by Renato Spigler

Received on 11 22, 2018. Accepted on 12 20, 2018.

\begin{abstract}
Chebyshev polynomials are traditionally applied to the approximation theory where are used in polynomial interpolation and also in the study of differential equations, in particular in some special cases of Sturm-Liouville differential equation. Many of the operational techniques presented, by using suitable integral transforms, via a symbolic approach to the Laplace transform, allow us to introduce polynomials recognized belonging to the families of Chebyshev of multi-dimensional type. The non-standard approach come out from the theory of multi-index Hermite polynomials, in particular by using the concepts and the related formalism of translation operators.
\end{abstract}

Keywords: Translation operators; Hermite polynomials; Generating functions; Chebyshev polynomials; Gegenbauer polynomials

AMS subject classification: 33C45, 33C47, 22E15, 33C50

\section{Introduction}

In order to better present the properties and the related operational relations that we will discuss for Hermite and Chebyshev polynomials, it is appropriate to highlight some important operational techniques that involve exponential operators [1].

We start to consider a real function $f(x)$, which is analytic in a neighborhood of the origin (it is easy to generalize to the complex case), so that can be expanded in Taylor series, in particular we can write:

$$
f(x+\lambda)=\sum_{n=0}^{+\infty} \frac{\lambda^{n}}{n !} f^{(n)}(x)
$$

where $\lambda$ is a continuous parameter.

The so called shift or translation operator $e^{\lambda \frac{d}{d x}}$, acts on the function $f(x)$ by producing a shift of the variable $x$ by the parameter $\lambda$ :

$$
e^{\lambda \frac{d}{d x}} f(x)=\sum_{n=0}^{+\infty} \frac{\lambda^{n}}{n !} \frac{d^{n}}{d x^{n}} f(x)=\sum_{n=0}^{+\infty} \frac{\lambda^{n}}{n !} f^{(n)}(x)
$$

and then from (1), gives:

$$
e^{\lambda \frac{d}{d x}} f(x)=f(x+\lambda)
$$

The concepts and the related formalism of exponential operators help us to derive many relevant relations involving analytic functions. In fact, it is easy to prove the following identities:

$$
e^{\lambda x \frac{d}{d x}} f(x)=f\left(e^{\lambda} x\right)
$$


where $\lambda$ is a continuous parameter,

$$
e^{\lambda x^{2} \frac{d}{d x}} f(x)=f\left(\frac{x}{1-\lambda x}\right)
$$

where $\lambda$ is a continuous parameter and $|x|<\frac{1}{|\lambda|}$,

$$
e^{\lambda x^{n} \frac{d}{d x}} f(x)=f\left(\frac{x}{n-1 \sqrt{1-(n-1) \lambda x^{n-1}}}\right)
$$

where $|x|<\left(\sqrt[n-1]{\frac{1}{(n-1)|\lambda|}}\right)$ and $\lambda$ continuous parameter. To generalize the action of the shift operator we look at the operator of the form:

$$
e^{\lambda q(x) \frac{d}{d x}}
$$

where $\lambda$ is a continuous parameter and the function $q(x)$ must satisfy some properties; in particular it is possible to prove the following relation:

$$
e^{\lambda q(x) \frac{d}{d x}} f(x)=f\left(\varphi\left(\varphi^{-1}(x)+\lambda\right)\right)
$$

where $\varphi(\theta)$ is a real function, which is invertible in a neighborhood of the origin and satisfies the identity:

$$
\varphi^{\prime}(\theta)=q(\varphi(\theta))
$$

This result can be used to define a more complicated shift operator; under the same hypothesis, we can, in fact introduce the following operator:

$$
E(x ; \lambda):=e^{\lambda\left(v(x)+q(x) \frac{d}{d x}\right)}
$$

such that:

$$
e^{\lambda\left(v(x)+q(x) \frac{d}{d x}\right)} x=x(\lambda) g(\lambda)
$$

where the function $x(\lambda)$ and $g(\lambda)$ must satisfy the following system of first order differential equations:

$$
\left\{\begin{array}{cc}
\frac{d}{d \lambda} x(\lambda)=q(x(\lambda)), & x(0)=x_{0} \\
\frac{d}{d \lambda} g(\lambda)=v(x(\lambda)) g(\lambda), & g(0)=1
\end{array}\right.
$$

Moreover, the identity (11) can be generalized to obtain the formula:

$$
e^{\lambda\left(v(x)+q(x) \frac{d}{d x}\right)} x^{n}=(x(\lambda))^{n} g(\lambda)
$$

and then, we can state the general statement:

$$
e^{\lambda\left(v(x)+q(x) \frac{d}{d x}\right)} f(x)=f(x(\lambda)) g(\lambda)
$$

with $f(x)$ analytic real function. An important aspect related to exponetial operators is represented by the disentangling rules [2], [3]. We start to note that in general, the exponential of two operators $\widehat{A}$ and $\widehat{B}$ does not satisfy the identity:

$$
e^{\widehat{A}+\widehat{B}}=e^{\widehat{A}} e^{\widehat{B}}
$$

as to the scalar case. There are many results which allow to calculate the compensation between the first and second member of the above relation, by using for instance the value of the commutator of the operators:

$$
[\widehat{A}, \widehat{B}]=\widehat{A} \widehat{B}-\widehat{B} \widehat{A}
$$


we can have, for a real parameter $\lambda$ and an analytic function $f(x)$, the relation

$$
e^{\lambda\left(x+\frac{d}{d x}\right)}=e^{\frac{\lambda^{2}}{2}} e^{\lambda x} e^{\lambda \frac{d}{d x}}
$$

It is easy to note that the above result can be generalized given the fundamental statement, noted as Weyl identity: for $\widehat{A}$ and $\widehat{B}$ two generic operators such that:

$$
\begin{gathered}
{[\widehat{A}, \widehat{B}]=k} \\
{[k, \widehat{A}]=[k, \widehat{B}]=0}
\end{gathered}
$$

where $k$ is the commutator, usually a real number, we have:

$$
e^{\widehat{A}+\widehat{B}}=e^{-\frac{k}{2}} e^{\widehat{A}} e^{\widehat{B}}
$$

A further important relation is the so-called Hausdorff identity:

$$
e^{\lambda \widehat{A}} \widehat{B} e^{-\lambda \widehat{A}}=\widehat{B}+\lambda[\widehat{A}, \widehat{B}]+\frac{\lambda^{2}}{2 !}[\widehat{A},[\widehat{A}, \widehat{B}]]+\frac{\lambda^{3}}{3 !}[\widehat{A},[\widehat{A},[\widehat{A}, \widehat{B}]]]+\ldots
$$

where $\widehat{A}$ and $\widehat{B}$ are two generic operators independent to the parameter $\lambda$.

The Hausdorff identity can be used in many applications, we can note for example, that $\forall m \in \mathbb{N}$ the following identity holds:

$$
e^{\lambda \frac{d^{m}}{d x^{m}}}(1)=\sum_{k=0}^{+\infty} \frac{\lambda^{k}}{k !} \frac{d^{k m}}{d x^{k m}}(1)=1
$$

and in particular for $m=2$, we have:

$$
e^{\lambda \frac{d^{2}}{d x^{2}}} x=\left(e^{\lambda \frac{d^{2}}{d x^{2}}} x e^{-\lambda \frac{d^{2}}{d x^{2}}}\right) e^{\lambda \frac{d^{2}}{d x^{2}}}(1)=e^{\lambda \frac{d^{2}}{d x^{2}}} x e^{-\lambda \frac{d^{2}}{d x^{2}}}(1)
$$

Moreover it is easy to obtain the following relevant relations:

$$
\begin{gathered}
e^{\lambda \frac{d^{2}}{d x^{2}}} x=\left(x+2 \lambda \frac{d}{d x}\right)(1)=x \\
e^{\lambda \frac{d^{2}}{d x^{2}}} x^{k}=\left(x+2 \lambda \frac{d}{d x}\right)^{k}(1)=x^{k}
\end{gathered}
$$

and finally, the Hausdorff identity can be used also for an analytic function $f(x)$, obtained:

$$
e^{\lambda \frac{d^{2}}{d x^{2}}} f(x)=f\left(x+2 \lambda \frac{d}{d x}\right)(1)
$$

and by choosing $f(x)=e^{x}$ (using the Weyl identity):

$$
e^{\lambda \frac{d^{2}}{d x^{2}}} e^{x}=e^{x+2 \lambda \frac{d}{d x}}(1)=e^{\lambda} e^{x} e^{2 \lambda \frac{d}{d x}}(1)=e^{\lambda+x}
$$

since:

$$
\left[x, 2 \lambda \frac{d}{d x}\right]=-2 \lambda
$$

We can also note that the relation (21) can be deduced from the general definition:

$$
e^{\lambda \frac{d^{m}}{d x^{m}}} e^{x}=\sum_{k=0}^{+\infty} \frac{\lambda^{k}}{k !} \frac{d^{k m}}{d x^{k m}} e^{x}=\sum_{k=0}^{+\infty} \frac{\lambda^{k}}{k !} e^{x}=e^{\lambda+x}
$$

which holds $\forall m \in \mathbb{N}$. 


\section{Generalized Hermite polynomials and relevant relations}

To introduce the generalized two-variable Hermite polynomials of the Gould-Hopper type [4], [5], we can use the formalism and the techniques of the exponential operators discussed in the previous section. By noting that:

$$
e^{y D} f(x)=f(x+y)=\sum_{n=0}^{+\infty} \frac{y^{n}}{n !} f^{(n)}(x)
$$

it is possible to generalize the procedure by considering the second derivative, obtaining:

$$
e^{y D^{2}} f(x)=\sum_{n=0}^{+\infty} \frac{y^{n}}{n !} f^{(2 n)}(x)
$$

and by noting that:

$$
D^{2 n} x^{m}=\frac{m !}{(m-2 n) !} x^{m-2 n}
$$

we have:

$$
e^{y D^{2}} x^{m}=\sum_{n=0}^{\left[\frac{m}{2}\right]} \frac{y^{n}}{n !} \frac{m !}{(m-2 n) !} x^{m-2 n}
$$

The above identity shows the general action of the exponential operators, so that we introduce the generalized two-variable Hermite polynomials of Kampé de Fériet form [4], [6]:

$$
H_{n}(x, y)=\sum_{s=0}^{\left[\frac{n}{2}\right]} \frac{n !}{s !(n-2 s) !} y^{n} x^{m-2 n}
$$

It is important to note that, assuming $f(x)=\sum_{n=0}^{+\infty} a_{n} x^{n}$, we can obtain from (26), the identity:

$$
e^{y D^{2}} f(x)=\sum_{n=0}^{+\infty} a_{n} H_{n}(x, y)
$$

and it is also easy to observe, that an elementary form of this kind of Hermite polynomials can be obtained:

$$
H_{n}^{(1)}(x, y)=(x+y)^{n}
$$

which can also be recast in the form:

$$
e^{y D} f(x)=\sum_{n=0}^{+\infty} a_{n} H_{n}^{(1)}(x, y)
$$

We note that the two-variable Hermite polynomials $H_{n}(x, y)$ are linked to the ordinary Hermite polynomials by the following relation:

$$
H_{n}\left(x,-\frac{1}{2}\right)=H e_{n}(x)
$$

where:

$$
H e_{n}(x)=n ! \sum_{r=0}^{\left[\frac{n}{2}\right]} \frac{(-1)^{r} x^{n-2 r}}{r !(n-2 r) ! 2^{r}}
$$


It is also important to note that the Hermite polynomials $H_{n}(x, y)$ satisfy the relation:

$$
H_{n}(x, 0)=x^{n}
$$

The relevant results satisfied from Hermite polynomials useful for our purposes are essentially related to differential relations involving the different families of Hermite polynomials that we are treating. We start to prove that the polynomials $H_{n}(x, y)$ solve the following partial differential equation [7]:

$$
\frac{\partial^{2}}{\partial x^{2}} H_{n}(x, y)=\frac{\partial}{\partial y} H_{n}(x, y)
$$

In fact, by deriving separately with respect to $x$ and to $y$, in (29), we obtain:

$$
\begin{aligned}
\frac{\partial}{\partial x} H_{n}(x, y) & =n H_{n-1}(x, y) \\
\frac{\partial}{\partial y} H_{n}(x, y) & =n(n-1) H_{n-2}(x, y)
\end{aligned}
$$

From the first of the above relation, by deriving again with respect to $x$ and by noting the second relation, we end up with (36).

This result helps us to derive an important operational rule for the Hermite polynomials $H_{n}(x, y)$; in fact, by considering the differential equation (36) as linear ordinary in the variable $y$ and by reminding the (35), we can immediately state the following relation:

$$
H_{n}(x, y)=e^{y \frac{\partial^{2}}{\partial x^{2}} x^{n}}
$$

The generating function of the above Hermite polynomials can be stated in many ways [8], [9]. We first note that the polynomials $H_{n}(x, y)$ satisfy the following differential difference equation:

$$
\begin{aligned}
\frac{d}{d z} Y_{n}(z) & =a n Y_{n-1}(z)+b n(n-1) Y_{n-2}(z) \\
Y_{n}(0) & =\delta_{n, 0}
\end{aligned}
$$

where $a$ and $b$ are real numbers, and since the proof is obtained by using the generating function method, setting:

$$
G(z ; t)=\sum_{n=0}^{+\infty} \frac{t^{n}}{n !} Y_{n}(z)
$$

with $t$ continuous variable, it is easy to obtain the relation linking the Hermite polynomials and their generating function:

$$
\exp \left(x t+y t^{2}\right)=\sum_{n=0}^{+\infty} \frac{t^{n}}{n !} H_{n}(x, y)
$$

We have introduced the two-variable Hermite polynomials $H_{n}(x, y)$ by using the concepts and the formalism of the translation operator. More in general the above Hermite polynomials can be derived, as a particular case, from a more general class of polynomials recognized as belonging to the Hermite family [10].

It is well known that the Hermite polynomials of the type $H_{n}^{(m)}(x, y)$ are defined by the formula:

$$
H_{n}^{(m)}(x, y)=\sum_{s=0}^{\left[\frac{n}{m}\right]} \frac{n !}{s !(n-m s) !} y^{s} x^{n-m s}
$$


It is easy to recognize that the above definition comes from relation (28), in fact, by noting that:

$$
e^{y D^{m}} f(x)=\sum_{n=0}^{+\infty} \frac{y^{n}}{n !} f^{(m n)}(x)
$$

and

$$
D^{m s} x^{n}=n(n-1) \ldots(n-m s+1) x^{n-m s}=\frac{n !}{(n-m s) !} x^{n-m s}
$$

for $s=0,1, \ldots\left[\frac{n}{m}\right]$ we obtain:

$$
e^{y D^{m}} x^{n}=\sum_{s=0}^{\left[\frac{n}{m}\right]} \frac{y^{s}}{s !} \frac{n !}{(n-m s) !} x^{n-m s}
$$

It also interesting to note that the Hermite polynomials $H_{n}^{(m)}(x, y)$ can also be introduced using directly their generating function: by exploiting the exponential $\exp \left(x t+y t^{m}\right)$ we can immediately recognize the identity:

$$
e^{x t+y t^{m}}=\sum_{n=0}^{+\infty} \frac{t^{n}}{n !} H_{n}^{(m)}(x, y)
$$

and then by setting $m=2$ we obtain the generating function of the Hermite polynomials $H_{n}(x, y)$. Similarly, these Hermite polynomials solved the following partial differential equation [7], [10]:

$$
\frac{\partial}{\partial y} H_{n}^{(m)}(x, y)=\frac{\partial^{m}}{\partial x^{m}} H_{n}^{(m)}(x, y)
$$

In fact, from (46), by differentiating with respect to $y$, we find:

$$
\sum_{n=0}^{+\infty} \frac{t^{n+m}}{n !} H_{n}^{(m)}(x, y)=\sum_{n=0}^{+\infty} \frac{t^{n}}{n !} \frac{\partial}{\partial y} H_{n}^{(m)}(x, y)
$$

after manipulating the l.h.s. of the above equation and by equating the like $t$ powers, we can immediately write:

$$
\frac{n !}{(n-m) !} H_{n-m}^{(m)}(x, y)=\frac{\partial}{\partial y} H_{n}^{(m)}(x, y)
$$

Otherwise, by deriving $m$-times with respect to $x$ in the (46), we have:

$$
\begin{aligned}
\frac{\partial}{\partial x} H_{n}^{(m)}(x, y) & =n H_{n-1}^{(m)}(x, y) \\
\frac{\partial^{m}}{\partial x^{m}} H_{n}^{(m)}(x, y) & =\frac{n !}{(n-m) !} H_{n-m}^{(m)}(x, y)
\end{aligned}
$$

and then by comparing the second equation of (50) with equation (49), we immediately obtain the partial differential equation (47).

The above result allows us to derive a similar operational definition for the Hermite polynomials $H_{n}^{(m)}(x, y)$ as in the case of the two-variable Kampé de Fériet polynomials. We note in fact that for $y=0$ in equation (42), we have:

$$
H_{n}^{(m)}(x, 0)=x^{n}
$$


and then, by considering the equation in (47) an ordinary differential equation in the variable $y$, we can immediately conclude that, since it is linear and of the first order, the solution can be expressed as:

$$
H_{n}^{(m)}(x, y)=e^{y \frac{\partial^{m}}{\partial x^{m}}} x^{n}
$$

or, in more explicit terms:

$$
H_{n}^{(m)}(x, y)=\left[\sum_{s=0}^{\left[\frac{n}{m}\right]} \frac{y^{r}}{s !}\left(\frac{\partial}{\partial x}\right)^{m s}\right] x^{n}
$$

\section{Special classes of Chebyshev polynomials and related integral representations}

We have introduced different type of generalized two-variable Hermite polynomials by using the techniques of the translation operator comparing with the method of the generating function. We now discuss the ordinary Chebyshev polynomials of the first and second kind, and we deal with their integral representations by using the concepts and operational techniques of the Hermite polynomials. Not only that, the use of Hermite polynomials makes it possible to establish new relationships and to introduce further families of generalized Chebyshev polynomials.

We remind that the ordinary first kind Chebyshev polynomials can be defined by the following relation [11], [12]:

$$
T_{n}(x)=\cos (n \arccos (x))
$$

with $x$ a real variable and the second kind Chebyshev polynomials through the relation:

$$
U_{n}(x)=\frac{\sin [(n+1) \arccos (x)]}{\sqrt{1-x^{2}}}
$$

The study of the properties of the Chebyshev polynomials can be simplified by introducing the following complex quantity [12]:

$$
\mathbf{T}_{n}(x)=\exp [i n(\arccos (x)]
$$

so that:

$$
\begin{aligned}
& \operatorname{Re}\left[\mathbf{T}_{n}(x)\right]=\cos (n \arccos (x)) \\
& \operatorname{Im}\left[\mathbf{T}_{n}(x)\right]=\sin (n \arccos (x))
\end{aligned}
$$

The above relations can be recast directly in terms of Chebyshev polynomials of the first and second kind. In fact, by noting that the second kind Chebyshev polynomials of degree $n-1$ reads:

$$
U_{n-1}(x)=\frac{\sin (n \arccos (x))}{\sqrt{1-x^{2}}}
$$

we can immediately conclude with:

$$
\begin{aligned}
T_{n}(x) & =\operatorname{Re}\left[\mathbf{T}_{n}(x)\right] \\
U_{n-1}(x) & =\frac{\operatorname{Im}\left[\mathbf{T}_{n}(x)\right]}{\sqrt{1-x^{2}}}
\end{aligned}
$$

To derive the related generating functions of the Chebyshev polynomials of the first and second kind, we can consider the generating function of the complex quantity, introduced in (56); let, in fact, be the real number $\xi$, such that $|\xi|<1$, we write:

$$
\sum_{n=0}^{+\infty} \xi^{n} \mathbf{T}_{n}(x)=\sum_{n=0}^{+\infty}\left(\xi e^{i \arccos (x)}\right)^{n}=\frac{1}{1-\xi e^{i \arccos (x)}}
$$


and then, it is immediately to state the following relations, involving the ordinary Chebyshev polynomials of first and second kind and their generating functions:

$$
\sum_{n=0}^{+\infty} \xi^{n} T_{n}(x)=\frac{1-\xi x}{1-2 \xi x+\xi^{2}}
$$

and

$$
\sum_{n=0}^{+\infty} \xi^{n} U_{n-1}(x)=\frac{\xi}{1-2 \xi x+\xi^{2}}
$$

where again, $\xi \in \mathbb{R}$, such that $|\xi|<1$.

We start to investigate the integral form of the ordinary Chebyshev polynomials, by using the concepts and the operational techniques of Hermite polynomials discussed before. We have introduced the second kind Chebyshev polynomials $U_{n}(x)$ through equation (55), so that we can immediately get the follow explicit form:

$$
U_{n}(x)=\sum_{k=0}^{\left[\frac{n}{2}\right]} \frac{(-1)^{k}(n-k) !(2 x)^{n-2 k}}{k !(n-2 k) !}
$$

The second kind Chebyshev polynomials satisfy the following integral representation [12], [13]:

$$
U_{n}(x)=\frac{1}{n !} \int_{0}^{+\infty} e^{-t} t^{n} H_{n}\left(2 x,-\frac{1}{t}\right) d t
$$

In fact, by noting that:

$$
n !=\int_{0}^{+\infty} e^{-t} t^{n} d t
$$

for $k \leq n$, we can write:

$$
(n-k) !=\int_{0}^{+\infty} e^{-t} t^{n-k} d t
$$

and from the explicit form of the Chebyshev polynomials $U_{n}(x)$, by recalling the standard form of the two-variable Hermite polynomials:

$$
H_{n}(x, y)=n ! \sum_{k=0}^{\left[\frac{n}{2}\right]} \frac{y^{k} x^{n-2 k}}{k !(n-2 k) !}
$$

we have, after a substitution and a manipulation:

$$
U_{n}(x)=\int_{0}^{+\infty} e^{-t} t^{n} \sum_{k=0}^{\left[\frac{n}{2}\right]} \frac{(-1)^{k} t^{-k}(2 x)^{n-2 k}}{k !(n-2 k) !} d t
$$

and then the statement.

By following the same procedure, we can also obtain an analogous integral representation for the Chebyshev polynomials of the first kind $T_{n}(x)$. In fact, it is easy to derive their explicit form:

$$
T_{n}(x)=\frac{n}{2} \sum_{k=0}^{\left[\frac{n}{2}\right]} \frac{(-1)^{k}(n-k-1) !(2 x)^{n-2 k}}{k !(n-2 k) !}
$$


and then, by using the same relations written above, we have:

$$
T_{n}(x)=\frac{1}{2(n-1) !} \int_{0}^{+\infty} e^{-t} t^{n-1} H_{n}\left(2 x,-\frac{1}{t}\right) d t
$$

These integral representations confirm the powerful tool represented by the Hermite polynomials. Moreover, the use of the previous discussed classes of Hermite polynomials makes it possible to estabilish relevant relations and introduce families of Chebyshev polynomials; we have in fact an important result linking the Chebyshev polynomials of the first and second kind.

Theorem 3.1. The Chebyshev polynomials $T_{n}(x)$ and $U_{n}(x)$ satisfy the following recurrence relations:

$$
\begin{aligned}
\frac{d}{d x} U_{n}(x) & =n W_{n-1}(x) \\
U_{n+1}(x) & =x W_{n}(x)-\frac{n}{n+1} W_{n-1}(x)
\end{aligned}
$$

and:

$$
T_{n+1}(x)=x U_{n}(x)-U_{n-1}(x)
$$

where:

$$
W_{n}(x)=\frac{2}{(n+1) !} \int_{0}^{+\infty} e^{-t} t^{n+1} H_{n}\left(2 x,-\frac{1}{t}\right) d t
$$

Proof. We note that the recurrence relations for the standard Hermite polynomials $H_{n}(x, y)$, can be costumized in the form [7]:

$$
\begin{aligned}
{\left[(2 x)+\left(-\frac{1}{t}\right) \frac{\partial}{\partial x}\right] H_{n}\left(2 x,-\frac{1}{t}\right) } & =H_{n+1}\left(2 x,-\frac{1}{t}\right) \\
\frac{1}{2} \frac{\partial}{\partial x} H_{n}\left(2 x,-\frac{1}{t}\right) & =n H_{n-1}\left(2 x,-\frac{1}{t}\right)
\end{aligned}
$$

After noting that expression of the integral representations (equations (64) and (67)) we obtain:

$$
\frac{d}{d x} U_{n}(x)=\frac{2 n}{n !} \int_{0}^{+\infty} e^{-t} t^{n} H_{n-1}\left(2 x,-\frac{1}{t}\right) d t
$$

and:

$$
\frac{d}{d x} T_{n}(x)=\frac{n}{(n-1) !} \int_{0}^{+\infty} e^{-t} t^{n-1} H_{n-1}\left(2 x,-\frac{1}{t}\right) d t
$$

Finally, since:

$$
U_{n-1}(x)=\frac{1}{(n-1) !} \int_{0}^{+\infty} e^{-t} t^{n-1} H_{n-1}\left(2 x,-\frac{1}{t}\right) d t
$$

we immediately get:

$$
\frac{d}{d x} T_{n}(x)=n U_{n-1}(x)
$$

Furthermore, by applying the Hermite multiplication operator to the second kind Chebyshev polynomials, we have:

$$
U_{n+1}(x)=\frac{1}{(n+1) !} \int_{0}^{+\infty} e^{-t} t^{n+1}\left[(2 x)+\left(-\frac{1}{t}\right) \frac{\partial}{\partial x}\right] H_{n}\left(2 x,-\frac{1}{t}\right) d t
$$




\section{Cesarano}

that is:

$$
\begin{gathered}
U_{n+1}(x)= \\
=x \frac{2}{(n+1) !} \int_{0}^{+\infty} e^{-t} t^{n+1} H_{n}\left(2 x,-\frac{1}{t}\right) d t-\frac{n}{n+1} \frac{2}{n !} \int_{0}^{+\infty} e^{-t} t^{n} H_{n-1}\left(2 x,-\frac{1}{t}\right) d t
\end{gathered}
$$

The second member of the r.h.s. of the above relation suggests us to introduce the following polynomials [12]:

$$
W_{n}(x)=\frac{2}{(n+1) !} \int_{0}^{+\infty} e^{-t} t^{n+1} H_{n}\left(2 x,-\frac{1}{t}\right) d t
$$

recognized as belonging to the families of the Chebyshev polynomials. Thus, from relation (71), we have:

$$
\frac{d}{d x} U_{n}(x)=n W_{n-1}(x)
$$

and, from identity (74), we get:

$$
U_{n+1}(x)=x W_{n}(x)-\frac{n}{n+1} W_{n-1}(x)
$$

Finally, by using the Hermite multiplication operator for the first kind Chebyshev polynomials, we can write:

$$
T_{n+1}(x)=\frac{1}{2 n !} \int_{0}^{+\infty} e^{-t} t^{n}\left[(2 x)+\left(-\frac{1}{t}\right) \frac{\partial}{\partial x}\right] H_{n}\left(2 x,-\frac{1}{t}\right) d t
$$

and then, after expanding the r.h.s. of the above relation, we can find:

$$
T_{n+1}(x)=x U_{n}(x)-U_{n-1}(x)
$$

which completely proves the theorem 3.1.

We start to generalize the ordinary Chebyshev polynomials by using the link with their integral representations. We note that for the second kind Chebyshev polynomials $U_{n}(x)$, it is also possible to state a different representation: in fact, by using their explicit form stated in (63), we can immediately write:

$$
U_{n}(x)=\frac{1}{n !} \int_{0}^{+\infty} e^{-t} H_{n}(2 x t,-t) d t
$$

that can be used to introduce the following generalization [13]:

$$
U_{n}(x, y ; \alpha)=\frac{1}{n !} \int_{0}^{+\infty} e^{-\alpha t} H_{n}(2 x t,-y t) d t
$$

where $x, y$ are real variables and $\alpha$ a real parameter.

By using the recurrence relations related to the two-variable Hermite polynomials, we can state the following expressions for the polynomials $U_{n}(x, y ; \alpha)$ :

$$
\begin{aligned}
\frac{\partial}{\partial y} U_{n}(x, y ; \alpha) & =\frac{\partial}{\partial \alpha} U_{n-2}(x, y ; \alpha) \\
\frac{\partial}{\partial x} U_{n}(x, y ; \alpha) & =-2 \frac{\partial}{\partial \alpha} U_{n-1}(x, y ; \alpha)
\end{aligned}
$$


The proof follows easily by noting that, once we derive with respect to $y$ in relation (81), we get:

$$
\frac{\partial}{\partial y} U_{n}(x, y ; \alpha)=\frac{1}{n !} \int_{0}^{+\infty} e^{-\alpha t} \frac{\partial}{\partial y} H_{n}(2 x t,-y t) d t
$$

and since:

$$
\frac{\partial}{\partial y} H_{n}(2 x t,-y t)=(-t) n(n-1) H_{n-2}(2 x t,-y t)
$$

we have:

$$
\frac{\partial}{\partial y} U_{n}(x, y ; \alpha)=\frac{1}{n !} \int_{0}^{+\infty} e^{-\alpha t}(-t) n(n-1) H_{n-2}(2 x t,-y t) d t
$$

which gives the first of (82).

In the same way, by noting that:

$$
\frac{\partial}{\partial x} H_{n}(2 x t,-y t)=(-2 t) n H_{n-1}(2 x t,-y t)
$$

we can derive the second of the equations displayed equation (82).

These recurrence relations allow us to prove the following result: the polynomials $U_{n}(x, y ; \alpha)$ satisfy the Cauchy problem:

$$
\left\{\begin{array}{c}
\frac{\partial^{2}}{\partial x^{2}} U_{n}(x, y ; \alpha)=-4 \frac{\partial^{2}}{\partial \alpha \partial y} U_{n}(x, y ; \alpha) \\
U_{n}(x, 0 ; \alpha)=\frac{(2 x)^{n}}{\alpha^{n+1}}
\end{array}\right.
$$

In fact, by deriving with respect to $x$ in the second of the equations stated in (82), we find:

$$
\frac{\partial^{2}}{\partial x^{2}} U_{n}(x, y ; \alpha)=-4 \frac{\partial}{\partial \alpha}\left(\frac{\partial}{\partial \alpha} U_{n-2}(x, y ; \alpha)\right)
$$

and then, since:

$$
\frac{\partial}{\partial \alpha} U_{n-2}(x, y ; \alpha)=\frac{\partial}{\partial y} U_{n}(x, y ; \alpha)
$$

we obtain:

$$
\frac{\partial^{2}}{\partial x^{2}} U_{n}(x, y ; \alpha)=-4 \frac{\partial^{2}}{\partial \alpha \partial y} U_{n}(x, y ; \alpha)
$$

Furthermore, by setting $y=0$ in relation (81), we have:

$$
U_{n}(x, 0 ; \alpha)=\frac{1}{n !} \int_{0}^{+\infty} e^{-\alpha t} H_{n}(2 x t, 0) d t
$$

and since:

$$
H_{n}(2 x t, 0)=(2 x t)^{n}
$$

we find:

$$
U_{n}(x, 0 ; \alpha)=\frac{(2 x)^{n}}{n !} \int_{0}^{+\infty} e^{-\alpha t} t^{n} d t
$$

that is:

$$
U_{n}(x, 0 ; \alpha)=\frac{(2 x)^{n}}{\alpha^{n+1}}
$$

The partial differential equation, stated in (83), can be viewed as a first order linear ordinary differential equation for the variable $y$, so that, by using the initial condition given by (85), we can state the solution:

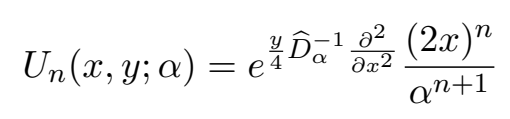




\section{Cesarano}

We note that the symbol $\widehat{D}_{\alpha}^{-1}$ denotes the inverse of the derivative [14], [15], [16]. By using the integral representation stated for the polynomials $U_{n}(x)$, and the related representation for the Chebyshev polynomials of the first kind $T_{n}(x)$ and the polynomials $W_{n}(x)$, we can easily introduce the following generalizations [13]:

$$
T_{n}(x, y ; \alpha)=\frac{1}{2(n-1) !} \int_{0}^{+\infty} e^{-\alpha t} t^{n-1} H_{n}\left(2 x,-\frac{y}{t}\right) d t
$$

and:

$$
W_{n}(x, y ; \alpha)=\frac{1}{(n+1) !} \int_{0}^{+\infty} e^{-\alpha t} t^{n+1} H_{n}\left(2 x,-\frac{y}{t}\right) d t
$$

With the above integral relations, it is immediate to state the following recurrence relations for these type of generalized Chebyshev polynomials:

$$
\begin{aligned}
\frac{\partial}{\partial \alpha} U_{n}(x, y ; \alpha) & =-\frac{1}{2}(n+1) W_{n}(x, y ; \alpha) \\
\frac{\partial}{\partial \alpha} T_{n}(x, y ; \alpha) & =-\frac{n}{2} U_{n}(x, y ; \alpha)
\end{aligned}
$$

For the first of the above equations the proof follows directly by deriving with respect to $\alpha$ in relation (87) and using equation (89), where we find:

$$
\frac{\partial}{\partial \alpha} U_{n}(x, y ; \alpha)=-\frac{1}{n !} \int_{0}^{+\infty} e^{-\alpha t} t^{n+1} H_{n}\left(2 x,-\frac{y}{t}\right) d t
$$

By following the same procedure it is possible to prove the second equation displayed in (90).

Before to conclude this section, we want to focus on a special aspect linking different families of polynomials. It is worth noting, in fact, that the Chebyshev polynomials can be viewed as a particular case of the Gegenbauer polynomials. We note that, the $n$-th order Gegenbauer polynomials reads [11], [17]:

$$
C_{n}^{(\mu)}(x)=\frac{1}{\Gamma(\mu)} \sum_{k=0}^{\left[\frac{n}{2}\right]} \frac{(-1)^{k}(2 x)^{n-2 k} \Gamma(n-k+\mu)}{k !(n-2 k) !}
$$

where $x$ and $\mu$ are real variables and $\Gamma(\mu)$ is the Euler function.

By recalling the integral representation of the above Euler function [11]:

$$
\Gamma(\mu)=\int_{0}^{+\infty} e^{-t} t^{\mu-1} d t
$$

and by using the same arguments exploited for the Chebyshev case, we can state the integral representation for the Gegenbauer polynomials:

$$
C_{n}^{(\mu)}(x)=\frac{1}{n ! \Gamma(\mu)} \int_{0}^{+\infty} e^{-t} t^{n+\mu-1} H_{n}\left(2 x,-\frac{1}{t}\right) d t
$$

We can also generalized these polynomials by using their integral representation, having:

$$
C_{n}^{(\mu)}(x, y ; \alpha)=\frac{1}{n ! \Gamma(\mu)} \int_{0}^{+\infty} e^{-\alpha t} t^{n+\mu-1} H_{n}\left(2 x,-\frac{y}{t}\right) d t
$$


where $x, y$ are real variables and $\alpha$ is a real parameter.

The introduction of this kind of Gegenbauer polynomials through the integral representation, represents a very flexible tool, in fact it can be exploited to derive the related generating function:

$$
\sum_{n=0}^{+\infty} \xi^{n} C_{n}^{(\mu)}(x, y ; \alpha)=\frac{1}{\left[\alpha-2 x \xi+y \xi^{2}\right]^{\mu}}
$$

where $\xi \in \mathbb{R}$, such that $|\xi|<1$ and $\mu \neq 0$.

Further generalization involving different kinds of Chebyshev polynomials can be obtained by using the concepts and the related formalism of Hermite polynomials of type $H_{n}^{(m)}(x, y)$. We introduce the generalized, $m$-order, two-variable second kind Chebyshev polynomials, the polynomials defined by the relation [17]:

$$
{ }_{m} U_{n}(x, y ; \alpha)=\frac{1}{n !} \int_{0}^{+\infty} e^{-\alpha t} t^{n} H_{n}^{(m)}\left(m x,-\frac{y}{t^{m-1}}\right) d t
$$

where $x, y$ are real variables and $\alpha$ is a real parameter.

It is possible to derive their generating function by using the properties of the Hermite polynomials of type $H_{n}^{(m)}(x, y)$; we have, indeed:

$$
\sum_{n=0}^{+\infty} \xi^{n}\left[{ }_{m} U_{n}(x, y ; \alpha)\right]=\frac{1}{\alpha-m x \xi+y \xi^{m}}
$$

where $\xi \in \mathbb{R},|\xi|<1$.

Note that, by multiplying both sides of the relation (96) by $\xi^{n}$ and by summing up over $n$, we have:

$$
\sum_{n=0}^{+\infty} \xi^{n}\left[{ }_{m} U_{n}(x, y ; \alpha)\right]=\int_{0}^{+\infty} e^{-\alpha t} \sum_{n=0}^{+\infty} \frac{(\xi t)^{n}}{n !} H_{n}^{(m)}\left(m x,-\frac{y}{t^{m-1}}\right) d t
$$

and by noting that the generating function of the Hermite polynomials of the type $H_{n}^{(m)}(x, y)$, is:

$$
\sum_{n=0}^{+\infty} \frac{(\xi t)^{n}}{n !} H_{n}^{(m)}\left(m x,-\frac{y}{t^{m-1}}\right)=\exp \left[m x(\xi t)-y\left(t \xi^{m}\right)\right]
$$

we obtain, in equation (98):

$$
\sum_{n=0}^{+\infty} \xi^{n}\left[{ }_{m} U_{n}(x, y ; \alpha)\right]=\int_{0}^{+\infty} e^{-\alpha t} e^{\left(m x \xi-y \xi^{m}\right) t} d t
$$

which, once integrating over $t$, gives the statement.

Similarly, we can generalize the Gegenbauer polynomials, by setting:

$$
{ }_{m} C_{n}^{(\mu)}(x, y ; \alpha)=\frac{1}{n ! \Gamma(\mu)} \int_{0}^{+\infty} e^{-\alpha t} t^{n+\mu-1} H_{n}^{(m)}\left(m x,-\frac{y}{t^{m-1}}\right) d t
$$

where $x, y$ are real variables, $\alpha$ is a real parameter and $\mu \in \mathbb{R}, \mu>0$.

It is easy to state, by following the same procedure used for the polynomials ${ }_{m} U_{n}(x, y ; \alpha)$, the generating function of the above generalized Gegenbauer polynomials, we have in fact:

$$
\sum_{n=0}^{+\infty} \xi^{n}\left[{ }_{m} C_{n}^{(\mu)}(x, y ; \alpha)\right]=\frac{1}{\left(\alpha-m x \xi+y \xi^{m}\right)^{\mu}}
$$


where, again $\xi \in \mathbb{R},|\xi|<1$.

The generalization of the Gegenbauer polynomials was also given by Gould [5], so that the procedure here described can be considered complementary; in particular the using of the Hermite polynomials and their related properties provides benefits to derive known and unknown relations. For instance we can also use the Gegenbauer polynomials, introduced in equation (91) or (93) to find further links between the ordinary Chebyshev polynomials.

In fact, by setting $\mu=2$, in relation (93), we have:

$$
C_{n}^{(2)}(x)=\frac{1}{n !} \int_{0}^{+\infty} e^{-t} t^{n+1} H_{n}\left(2 x,-\frac{1}{t}\right) d t
$$

which can be easily recognized as:

$$
C_{n}^{(2)}(x)=\frac{n+1}{2} W_{n}(x)
$$

where the polynomials $W_{n}(x)$ have been specified in (75).

In this section we have seen interesting integral representations related to ordinary and generalized Chebyshev polynomials. The common denominator with respect to which were derived properties are the plethora of identities and operational relations satisfied by the Hermite polynomials in their different forms. In the next section, we will investigate families of polynomials that can be traced to the Chebyshev polynomials, always operating with the aid of the Hermite polynomials.

\section{Chebyshev-like polynomials and concluding remarks}

We now can discuss the two-variable Chebyshev polynomials by using the generalized two-variable Hermite polynomials and their integral representations concerning the ordinary and generalized Chebyshev polynomials.

We introduce the two-variable Chebyshev polynomials of the second kind, by setting [18]:

$$
U_{n}(x, y)=\sum_{k=0}^{\left[\frac{n}{2}\right]} \frac{(n-k) ! x^{n-2 k} y^{k}}{k !(n-2 k) !}
$$

where $x$ and $y$ are real variables. It is easy to note that the above polynomials can be derived directly from the explicit form of the standard second kind Chebyshev polynomials, or by using the integral representation of the polynomials $U_{n}(x, y ; \alpha)$. We have, in fact:

$$
U_{n}(x, y)=\frac{1}{n !} \int_{0}^{+\infty} e^{-t} t^{n} H_{n}\left(x, \frac{y}{t}\right) d t
$$

It is enough to note that:

$$
(n-k) !=\int_{0}^{+\infty} e^{-t} t^{n-k} d t
$$

which gives in relation (102):

$$
U_{n}(x, y)=\int_{0}^{+\infty} e^{-t} t^{n} \sum_{k=0}^{\left[\frac{n}{2}\right]} \frac{x^{n-2 k} y^{k} t^{-k}}{k !(n-2 k) !} d t
$$

and then the statement.

The above result helps us to state the link between the Chebyshev polynomials $U_{n}(x, y)$ and the slight different one-parameter Chebyshev polynomials $U_{n}(x, y ; \alpha)$ presented in the previous section, that is:

$$
U_{n}\left(\frac{x}{2},-y ; 1\right)=U_{n}(x, y)
$$


By following the above procedure, we can introduce the analogous generalization of the first kind Chebyshev polynomials through their integral representation:

$$
T_{n}(x, y)=\frac{1}{(n-1) !} \int_{0}^{+\infty} e^{-t} t^{n-1} H_{n}\left(x, \frac{y}{t}\right) d t
$$

and by using the same arguments exploited in the previous discussions, it is possible to derive some useful recurrence relations involving the generalized Chebyshev polynomials $U_{n}(x, y)$ and $T_{n}(x, y)$, that is

$$
2 y \frac{\partial}{\partial x} U_{n-1}(x, y)=\left(n-x \frac{\partial}{\partial x}\right) U_{n}(x, y)
$$

and:

$$
T_{n+1}(x, y)=x U_{n}(x, y)+2 y U_{n-1}(x, y)
$$

The above relations give the further recurrence:

$$
U_{n+1}(x, y)=x U_{n}(x, y)+y U_{n-1}(x, y)
$$

The recurrences (107) and (108) can be exploited to define rising and lowering operators for generalized Chebyshev polynomials; indeed, by using the operator $\widehat{D}_{x}^{-1}[14]$, denoting a kind of inverse derivative, we can immediately write:

$$
U_{n-1}(x, y)=\frac{1}{2 y} \widehat{D}_{x}^{-1}\left[n-x \frac{\partial}{\partial x}\right] U_{n}(x, y)
$$

and:

$$
U_{n+1}(x, y)=\left[x+\frac{1}{2} \widehat{D}_{x}^{-1}\left(n-x \frac{\partial}{\partial x}\right)\right] U_{n}(x, y)
$$

which define:

$$
\begin{gathered}
\widehat{E}_{+}=x+\frac{1}{2} \widehat{D}_{x}^{-1}\left(n-x \frac{\partial}{\partial x}\right) \\
\widehat{E}_{-}=\frac{1}{2 y} \widehat{D}_{x}^{-1}\left(n-x \frac{\partial}{\partial x}\right)
\end{gathered}
$$

whose action can be written as:

$$
\begin{aligned}
& \widehat{E}_{+} U_{n}(x, y)=U_{n+1}(x, y) \\
& \widehat{E}_{-} U_{n}(x, y)=U_{n-1}(x, y)
\end{aligned}
$$

The above operators can be exploited to derive the differential equation satisfied by the generalized two-variable Chebyshev polynomials $U_{n}(x, y)$.

Theorem 4.1. The polynomials $U_{n}(x, y)$ satisfy the following partial differential equation:

$$
\left[\left(4 y+x^{2}\right) \frac{\partial^{2}}{\partial x^{2}}+3 x \frac{\partial}{\partial x}-n(n+2)\right] U_{n}(x, y)=0
$$




\section{Cesarano}

Proof. By using the rising and the lowering operators defined in (113) and (114), we can immediately write:

$$
\widehat{E}_{-}\left[\widehat{E}_{+} U_{n}(x, y)\right]=U_{n}(x, y)
$$

which can be expanded to give:

$$
\frac{1}{2 y} \widehat{D}_{x}^{-1}\left[\left((n+1)-x \frac{\partial}{\partial x}\right)\right]\left[x+\frac{1}{2} \widehat{D}_{x}^{-1}\left(n-x \frac{\partial}{\partial x}\right)\right] U_{n}(x, y)=U_{n}(x, y)
$$

By noting that:

$$
\frac{\partial}{\partial x} \widehat{D}_{x}^{-1}=\widehat{1}
$$

we can derive with respect to $x$ in relation (117), obtained:

$$
\left[(n+1)-x \frac{\partial}{\partial x}\right]\left[x+\frac{1}{2} \widehat{D}_{x}^{-1}\left(n-x \frac{\partial}{\partial x}\right)\right] U_{n}(x, y)=2 y \frac{\partial}{\partial x} U_{n}(x, y)
$$

that is:

$$
\begin{gathered}
{\left[(n+1) x+\frac{n+1}{2} \widehat{D}_{x}^{-1}\left(n-x \frac{\partial}{\partial x}\right)-x \frac{\partial}{\partial x} x-\frac{1}{2} x\left(n-x \frac{\partial}{\partial x}\right)\right] U_{n}(x, y)=} \\
=2 y \frac{\partial}{\partial x} U_{n}(x, y)
\end{gathered}
$$

and again:

$$
\begin{gathered}
{\left[(n+1) \frac{\partial}{\partial x} x+\frac{n+1}{2}\left(n-x \frac{\partial}{\partial x}\right)-\frac{\partial}{\partial x} x \frac{\partial}{\partial x} x-\frac{1}{2} \frac{\partial}{\partial x} x\left(n-x \frac{\partial}{\partial x}\right)\right] U_{n}(x, y)=} \\
=2 y \frac{\partial^{2}}{\partial x^{2}} U_{n}(x, y)
\end{gathered}
$$

By noting that:

$$
x \frac{\partial}{\partial x}=\frac{\partial}{\partial x} x-1
$$

we can rewrite (120) in the form:

$$
\begin{gathered}
{\left[(n+1)+(n+1) x \frac{\partial}{\partial x}+\frac{n(n+1)}{2}-\frac{n+1}{2} x \frac{\partial}{\partial x}-\frac{\partial}{\partial x} x^{2} \frac{\partial}{\partial x}+\right.} \\
\left.-\frac{n}{2} \frac{\partial}{\partial x} x+\frac{1}{2} \frac{\partial}{\partial x} x^{2} \frac{\partial}{\partial x}\right] U_{n}(x, y)=2 y \frac{\partial^{2}}{\partial x^{2}} U_{n}(x, y)
\end{gathered}
$$

The above identity can be also recast in a more convenient form; indeed, by noting that:

$$
\frac{\partial}{\partial x} x^{2} \frac{\partial}{\partial x}=2 x \frac{\partial}{\partial x}+x^{2} \frac{\partial^{2}}{\partial x^{2}}
$$

we write:

$$
\begin{gathered}
\left\{-\frac{1}{2} x^{2} \frac{\partial^{2}}{\partial x^{2}}+\left[\frac{2(n+1)-(n+1)-4-n}{2}\right] x \frac{\partial}{\partial x}+\right. \\
\left.+\left[\frac{2(n+1)+n(n+1)-2-n}{2}\right]\right\} U_{n}(x, y)=2 y \frac{\partial^{2}}{\partial x^{2}} U_{n}(x, y)
\end{gathered}
$$


and finally:

$$
\left[-\frac{1}{2} x^{2} \frac{\partial^{2}}{\partial x^{2}}-\frac{3}{2} x \frac{\partial}{\partial x}+\frac{n(n+2)}{2}\right] U_{n}(x, y)=2 y \frac{\partial^{2}}{\partial x^{2}} U_{n}(x, y)
$$

which immediately gives theorem 4.1.

We now introduce a further generalizations of Chebyshev polynomials, could be defined as Chebyshevlike polynomials, by using the generalized $m$-order Hermite polynomials and we derive the related integral representations. The generalized two-variable, $m$-order Chebyshev polynomials are defined by the formula:

$$
U_{n}^{(m)}(x, y)=\sum_{k=0}^{\left[\frac{n}{m}\right]} \frac{(n-k) ! x^{n-m k} y^{k}}{k !(n-m k) !}
$$

where $x, y \in \mathbb{R}$ and $n, m \in \mathbb{N}$.

By using the Hermite polynomials $H_{n}^{(m)}(x, y)$ we can immediately derive the integral representation:

$$
U_{n}^{(m)}(x, y)=\frac{1}{n !} \int_{0}^{+\infty} e^{-t} t^{n} H_{n}^{(m)}\left(x, \frac{y}{t}\right) d t
$$

By using the relations verified from the Hermite polynomials and by following the same procedure used for the Chebyshev polynomials $U_{n}(x, y)$, we easily state the following recurrence relations:

$$
\begin{gathered}
m y \frac{\partial^{m-1}}{\partial x^{m-1}} U_{n-1}^{(m)}(x, y)=\left(n-x \frac{\partial}{\partial x}\right) U_{n}^{(m)}(x, y) \\
U_{n+1}^{(m)}(x, y)=x U_{n}^{(m)}(x, y)+(m-1) y \frac{\partial^{m-2}}{\partial x^{m-2}} U_{n-1}^{(m)}(x, y)
\end{gathered}
$$

These identities allow us to define the rising and lowering operators related to the generalized Chebyshev polynomials $U_{n}^{(m)}(x, y)$, by setting:

$$
\begin{gathered}
\widehat{E}_{+}=x+\frac{m-1}{m} \widehat{D}_{x}^{-1}\left(n-x \frac{\partial}{\partial x}\right) \\
\widehat{E}_{-}=\frac{1}{m y} \widehat{D}_{x}^{-(m-1)}\left(n-x \frac{\partial}{\partial x}\right)
\end{gathered}
$$

which, as we have noted before, act on the polynomials $U_{n}^{(m)}(x, y)$ as follows:

$$
\widehat{E}_{+} U_{n}^{(m)}(x, y)=U_{n+1}^{(m)}(x, y)
$$

which give the following important result.

Theorem 4.2. The polynomials $U_{n}^{(m)}(x, y)$ satisfy the following partial differential equation:

$$
\left[m y \frac{\partial^{m}}{\partial x^{m}}+\frac{x^{2}}{m} \frac{\partial^{2}}{\partial x^{2}}+\left(1+n-\frac{2 n-1}{m}\right) x \frac{\partial}{\partial x}-n\left(1+\frac{n(m-1)}{m}\right)\right] U_{n}^{(m)}(x, y)=0
$$


Proof. By noting that:

$$
\widehat{E}_{-} \widehat{E}_{+} U_{n}^{(m)}(x, y)=U_{n}^{(m)}(x, y)
$$

which in explicit forms, reads:

$$
\widehat{D}_{x}^{-(m-1)}\left((n+1)-x \frac{\partial}{\partial x}\right)\left[x+\frac{m-1}{m} \widehat{D}_{x}^{-1}\left(n-x \frac{\partial}{\partial x}\right)\right] U_{n}^{(m)}(x, y)=m y U_{n}^{(m)}(x, y)
$$

and since:

$$
\begin{aligned}
& \frac{\partial^{m-1}}{\partial x^{m-1}} \widehat{D}_{x}^{-(m-1)}=\widehat{1} \\
& \widehat{D}_{x}^{-(m-1)} \frac{\partial^{m-1}}{\partial x^{m-1}}=\widehat{1}
\end{aligned}
$$

by deriving $m$-times with respect to $x$ in equation (133), we obtain:

$$
\left((n+1)-x \frac{\partial}{\partial x}\right)\left[x+\frac{m-1}{m} \widehat{D}_{x}^{-1}\left(n-x \frac{\partial}{\partial x}\right)\right] U_{n}^{(m)}(x, y)=m y \frac{\partial^{m-1}}{\partial x^{m-1}} U_{n}^{(m)}(x, y)
$$

Finally, by noting that the following identities hold:

$$
\begin{aligned}
\frac{\partial}{\partial x} x & =1+x \frac{\partial}{\partial x} \\
\frac{\partial}{\partial x} x^{2} \frac{\partial}{\partial x} & =2 x \frac{\partial}{\partial x}+x^{2} \frac{\partial^{2}}{\partial x^{2}}
\end{aligned}
$$

we can rearranged identity (134) in the form:

$$
\begin{gathered}
m y \frac{\partial^{m}}{\partial x^{m}}=(n+1)\left(1+x \frac{\partial}{\partial x}\right)+\frac{(n+1)(m-1)}{m}\left(n-x \frac{\partial}{\partial x}\right)+ \\
-\frac{\partial}{\partial x} x\left(1+x \frac{\partial}{\partial x}\right)+\frac{n(m-1)}{m} \frac{\partial}{\partial x} x+\frac{m-1}{m} \frac{\partial}{\partial x} x^{2} \frac{\partial}{\partial x}
\end{gathered}
$$

and then:

$$
m y \frac{\partial^{m}}{\partial x^{m}}=-\frac{x^{2}}{m} \frac{\partial^{2}}{\partial x^{2}}+\left(-n-1+\frac{2 n-1}{m}\right) x \frac{\partial}{\partial x}+n\left(1+\frac{n(m-1)}{m}\right)
$$

which proves theorem 4.2 .

It has been outlined previously that the families of polynomials, called as Chebyshev-like polynomials, present a substantial generalization of the ordinary Chebyshev polynomials; a fortiori, the polynomials denoted through the relation:

$$
U_{n}^{(m)}(x, y)=\sum_{k=0}^{\left[\frac{n}{2}\right]} \frac{(n-k) ! x^{n-2 k} y^{k}}{k !(n-2 k) !}
$$

and the related properties, further show a different nature with respect to the ordinary Chebyshev polynomials, while on the contrary prove their similarity to the generalized Hermite polynomials of the form:

$$
H_{n}^{(m)}(x, y)=\sum_{s=0}^{\left[\frac{n}{m}\right]} \frac{n !}{s !(n-m s) !} y^{s} x^{n-m s}
$$

It is possible to derive further generalizations for families of polynomials recognized as Chebyshev-like polynomials, again with the formalism and the related operational techniques own by further classes of Hermite polynomials. More precisely, it is possible to proceed in a parallel manner in deriving new integral representations which involve Chebyshev-like polynomials and new families of polynomials attributable to the family of Hermite. This suggests that further progress can be made with respect to the integral representations which involve Chebyshev and Hermite polynomials of generalized type. 


\section{References}

1. B. M. Levitan, Generalized translation operators and some of their applications. Jerusalem: Israel Program for Scientific Translations, 1964.

2. Y. Smirnov and A. V. Turbiner, Hidden sl 2-algebra of finite-difference equations 1, Modern Physics Letters, vol. A10, pp. 1795-1801, 1995.

3. W. Miller, Lie theory and special functions. Academic Press, 1968.

4. P. Appell and J. K. de Fériet, Fonctions Hypergéométriques et Hypersphériques: Polynômes d'Hermite. Paris: Gauthier-Villars, 1926.

5. H. W. Gould, A. Hopper, et al., Operational formulas connected with two generalizations of Hermite polynomials, Duke Mathematical Journal, vol. 29, no. 1, pp. 51-63, 1962.

6. C. Cesarano, Operational methods and new identities for Hermite polynomials, Mathematical Modelling of Natural Phenomena, vol. 12, no. 3, pp. 44-50, 2017.

7. C. Cesarano, C. Fornaro, and L. Vazquez, A note on a special class of Hermite polynomials, International Journal of Pure and Applied Mathematics, vol. 98, no. 2, pp. 261-273, 2015.

8. H. Srivastava and H. Manocha, Treatise on generating functions. Ney York: John Wiley \& Sons, 1984.

9. C. Cesarano, G. M. Cennamo, and L. Placidi, Operational methods for Hermite polynomials with applications, WSEAS Transaction on Mathematics, vol. 13, pp. 925-931, 2014.

10. C. Cesarano, C. Fornaro, and L. Vazquez, Operational results in bi-orthogonal Hermite functions, Acta Mathematica Universitatis Comenianae, vol. 85, no. 1, pp. 43-68, 2016.

11. M. Abramowitz and I. A. Stegun, Handbook of mathematical functions: with formulas, graphs, and mathematical tables, vol. 55. Courier Corporation, 1965.

12. C. Cesarano, Generalized Chebyshev polynomials, Hacet. J. Math. Stat, vol. 43, no. 5, pp. 731-740, 2014.

13. C. Cesarano, Integral representations and new generating functions of Chebyshev polynomials, Rev. Mat. Complut, 2015.

14. C. Cesarano and P. Ricci, The Legendre polynomials as a basis for Bessel functions, International Journal of Pure and Applied Mathematics, vol. 111, no. 1, pp. 129-139, 2016.

15. G. Dattoli, Hermite-Bessel and Laguerre-Bessel functions: a by-product of the monomiality principle, 1999.

16. P. Ricci and I. Tavkhelidze, An introduction to operational techniques and special polynomials, Journal of mathematical sciences, vol. 157, no. 1, pp. 161-189, 2009.

17. C. Cesarano, Generalization of two-variable Chebyshev and Gegenbauer polynomials, International Journal of Applied Mathematics and Statistics, vol. 53, no. 1, pp. 1-7, 2015.

18. C. Cesarano and C. Fornaro, Operational identities on generalized two-variable Chebyshev polynomials, International Journal of Pure and Applied Mathematics, vol. 100, no. 1, pp. 59-74, 2015. 